\title{
Instanton Calculus With R-R Background And Topological Strings
}

\author{
Marco Billó ${ }^{1 *}$ \\ ${ }^{1}$ Dip. di Fisica Teorica, Università di Torino and I.N.FN., sez. di Torino \\ Via Pietro Giuria 1, 10125 Torino (ITALY)
}

\begin{abstract}
The non-perturbative low energy effective action of N=2 SYM is studied within a microscopic string realization via D3/D-instanton systems. The localization deformation of instanton moduli space which has allowed the exact computation of multi-instanton contributions is, in this setting, due to a RR graviphoton background. The relation of deformed instanton contributions to topological string amplitudes on CY, argued by Nekrasov, appears quite natural in this framework. Based on [1].
\end{abstract}

To appear in the proceedings of the RTN workshop "ForcesUniverse", Naples, October 9-13 2006

\section{Introduction}

Recently, D-instanton-induced interactions in the low energy effective actions are receiving quite some attention. In this contribution, we study D-instanton induced couplings of the chiral and the Weyl multiplet in $\mathcal{N}=2$ SYM low energy effective theory. In this framework, we obtain a natural interpretation of a conjecture by Nekrasov regarding the $\mathcal{N}=2$ multi-instanton calculus and its relation to topological string amplitudes on CY's

The semiclassical limit of the Seiberg-Witten prepotential displays 1-loop plus instanton contributions:

$$
\mathcal{F}(a)=\frac{\mathrm{i}}{2 \pi} a^{2} \log \frac{a^{2}}{\Lambda^{2}}+\sum_{k=1}^{\infty} \mathcal{F}^{(k)}(a) .
$$

After the Seiberg-Witten solution was put forward, it became very important to check it against the computation of the multi-instanton contributions $\mathcal{F}^{(k)}(a)$ within the "microscopic" description of the non-abelian gauge theory. The calculation of these coefficients has only recently been fully accomplished [2, 3, 4]. Introducing a suitable deformation of the ADHM measure on the super-instanton moduli spaces which exploits the $4 \mathrm{~d}$ chiral rotations symmetry of ADHM constraints, the resulting partition function can be computed using localization techniques and the topological twist of its supersymmetries. One has

$$
Z(a, \varepsilon)=\sum_{k} Z^{(k)}(a, \varepsilon)=\sum_{k} \int d \widehat{\mathcal{M}}_{(k)} \mathrm{e}^{-\mathcal{S}_{\bmod }\left(a, \varepsilon ; \mathcal{M}_{(k)}\right)}=\exp \left(\frac{\mathcal{F}_{\text {n.p. }}(a ; \varepsilon)}{\varepsilon^{2}}\right)
$$

and $\lim _{\varepsilon \rightarrow 0} \mathcal{F}_{\text {n.p. }}(a ; \varepsilon)=\mathcal{F}_{\text {n.p. }}(a)$ yields the non-perturbative part of the SW prepotential. What about higher orders in the deformation parameter $\varepsilon$ ?

Nekrasov proposed [2, 4, 5] that terms of order $\varepsilon^{2 h}$ correspond to gravitational $F$-terms in the $\mathcal{N}=2$ eff. action involving metric and graviphoton curvatures:

$$
\int d^{4} x\left(R^{+}\right)^{2}\left(\mathcal{F}^{+}\right)^{2 h-2}
$$

When the effective $\mathcal{N}=2$ theory is obtained from type II strings on a "local" CY manifold $\mathfrak{M}$ via geometrical engineering [6, 7], such terms arise from world-sheets of genus $h$ and are computed by the topological string [8,9]. For the local $\mathrm{CY}$ describing the $\mathrm{SU}(2)$ theory the proposal has been tested in [10].

* Corresponding author E-mail: billo@to.infn.it, Phone: +0039011 670 7213, Fax: +0039011 6707213 
Table 1 The spectrum of moduli for the fractional D(-1)/D3 system

\begin{tabular}{c|cccc} 
& ADHM & Meaning & Vertex & Chan-Paton \\
\hline$-1 /-1$ (NS) & $a_{\mu}^{\prime}$ & centers & $\psi^{\mu}(z) \mathrm{e}^{-\varphi(z)}$ & adj. U(k) \\
& $\chi$ & aux. & $\bar{\Psi}(z) \mathrm{e}^{-\varphi(z)}$ & $\vdots$ \\
(aux. vert.) & $D_{c}$ & Lagrange mult. & $\bar{\eta}_{\mu \nu}^{c} \psi^{\nu}(z) \psi^{\mu}(z)$ & $\vdots$ \\
(R) & $M^{\alpha A}$ & partners & $S_{\alpha}(z) S_{A}(z) \mathrm{e}^{-\frac{1}{2} \varphi(z)}$ & $\vdots$ \\
& $\lambda_{\dot{\alpha} A}$ & Lagrange mult. & $S^{\dot{\alpha}}(z) S^{A}(z) \mathrm{e}^{-\frac{1}{2} \varphi(z)}$ & $\vdots$ \\
\hline$-1 / 3$ (NS) & $w_{\dot{\alpha}}$ & sizes & $\Delta(z) S^{\dot{\alpha}}(z) \mathrm{e}^{-\varphi(z)}$ & $k \times \bar{N}$ \\
& $\bar{w}_{\dot{\alpha}}$ & $\vdots$ & $\bar{\Delta}(z) S^{\dot{\alpha}}(z) \mathrm{e}^{-\varphi(z)}$ & $\vdots$ \\
& $\mu^{A}$ & partners & $\Delta(z) S_{A}(z) \mathrm{e}^{-\frac{1}{2} \varphi(z)}$ & $\vdots$ \\
& $\bar{\mu}^{A}$ & $\vdots$ & $\bar{\Delta}(z) S_{A}(z) \mathrm{e}^{-\frac{1}{2} \varphi(z)}$ & $\vdots$ \\
\hline
\end{tabular}

We will discuss how to reproduce the semiclassical instanton expansion of the low energy effective action for the $\mathcal{N}=2 \mathrm{SYM}$ theory via its microscopic string realization via (fractional) $\mathrm{D} 3 / \mathrm{D}(-1)$ branes. We will then show that the inclusion of the graviphoton of the $\mathcal{N}=2$ bulk sugra, which comes from the RR sector, produces in the effective action the gravitational F-terms which are computed by the topological string on local CY; at the same time, it leads exactly to the localization deformation on the instanton moduli space which allows to perform the integration.

\section{Stringy instanton calculus for $\mathcal{N}=2 \mathbf{S Y M}$}

Consider pure $\mathrm{SU}(N)$ Yang-Mills in 4 dimensions with $\mathcal{N}=2$ susy. It is realized by the massless d.o.f. of open strings attached to fractional D3-branes in the orbifold background $\mathbb{R}^{4} \times \mathbb{R}^{2} \times \mathbb{R}^{4} / \mathbb{Z}_{2}$ These d.o.f. can be can be arranged in a $\mathcal{N}=2$ chiral superfield in the adjoint of $\mathrm{U}(N)$ :

$$
\Phi(x, \theta)=\phi(x)+\theta \Lambda(x)+\frac{1}{2} \theta \sigma^{\mu \nu} \theta F_{\mu \nu}^{+}(x)+\cdots
$$

String amplitudes for the string vertices corresponding to the SYM fields of Eq. (1) on discs attached to the D3 branes give rise, in the limit $\alpha^{\prime} \rightarrow 0$ with gauge quantities fixed, to the tree level (microscopic) $\mathcal{N}=2$ action for $\mathrm{U}(N) \mathrm{SYM}$, where $N$ is the number of fractional D3-branes. We are interested in the 1.e.e.a. on the Coulomb branch parametrized by the v.e.v.'s $\left\langle\Phi_{u v}\right\rangle=a_{u} \delta_{u v}$ of the adjoint chiral superfields breaking $\mathrm{SU}(N) \rightarrow \mathrm{U}(1)^{N-1}$; we focus for simplicity on $\mathrm{SU}(2)$. Up to two-derivatives, $\mathcal{N}=2$ susy forces the effective action for the chiral multiplet $\Phi$ in the Cartan direction to be of the form

$$
S_{\text {eff }}[\Phi]=\int d^{4} x d^{4} \theta \mathcal{F}(\Phi)+\text { c.c },
$$

where $\mathcal{F}$ is called the prepotential of the theory. We want now to discuss how the instanton corrections to the prepotential arise in our string set-up.

Since the topological density of an instantonic configuration corresponds to a localized source for the RR scalar $C_{0}$ in the WZ part of the D3-brane action, instanton-charge $k$ solutions of $3+1 \operatorname{dims} . \mathrm{SU}(N)$ gauge theories correspond to $k$ D-instantons inside $N$ D3-branes [11, 12]

Open strings ending on a $\mathrm{D}(-1)$ carry no momentum: the polarizations of their physical vertices are moduli (rather than fields), and they correspond to the parameters of the instanton, see Table 1 we will denote them as $\mathcal{M}_{(k)}$.

Let us consider first disc diagrams involving only moduli and no D3/D3 state; these are "vacuum" contributions from the D3 point of view; see Fig. 1]The combinatorics of boundaries [13] is such that these 


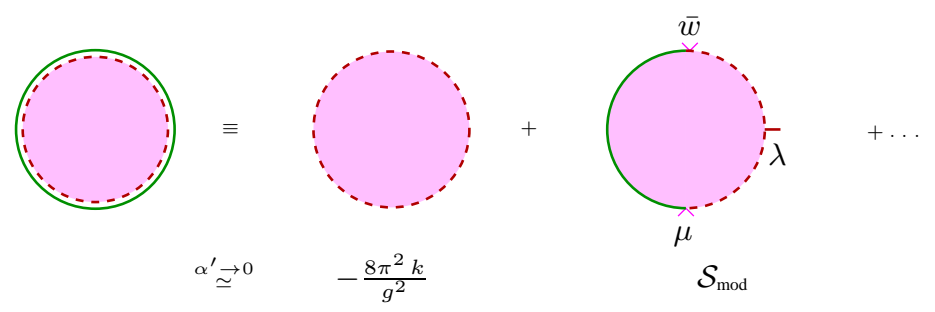

Fig. 1 D-instanton discs contributing to the partition function.

D-instanton diagrams exponentiate. Moreover, the moduli must be integrated over to produce the partition function

$$
Z^{(k)}=\int d \mathcal{M}_{(k)} \mathrm{e}^{-\frac{8 \pi^{2} k}{g^{2}}-\mathcal{S}_{\bmod }}
$$

From disc diagrams with insertion of moduli vertices, in the field theory limit we extract the ADHM moduli action (at fixed $k$ ): $\mathcal{S}_{\text {mod }}=\mathcal{S}_{\text {bos }}^{(k)}+\mathcal{S}_{\text {fer }}^{(k)}+\mathcal{S}_{\mathrm{c}}^{(k)}$, with

$$
\begin{aligned}
\mathcal{S}_{\text {bos }}^{(k)} & =\operatorname{tr}_{k}\left\{-2\left[\chi^{\dagger}, a_{\mu}^{\prime}\right]\left[\chi, a^{\prime \mu}\right]+\chi^{\dagger} \bar{w}_{\dot{\alpha}} w^{\dot{\alpha}} \chi+\chi \bar{w}_{\dot{\alpha}} w^{\dot{\alpha}} \chi^{\dagger}\right\}, \\
\mathcal{S}_{\text {fer }}^{(k)} & =\operatorname{tr}_{k}\left\{\mathrm{i} \frac{\sqrt{2}}{2} \bar{\mu}^{A} \epsilon_{A B} \mu^{B} \chi^{\dagger}-\mathrm{i} \frac{\sqrt{2}}{4} M^{\alpha A} \epsilon_{A B}\left[\chi^{\dagger}, M_{\alpha}^{B}\right]\right\}, \\
\mathcal{S}_{\mathrm{c}}^{(k)} & =\operatorname{tr}_{k}\left\{-\mathrm{i} D_{c}\left(W^{c}+\mathrm{i} \bar{\eta}_{\mu \nu}^{c}\left[a^{\prime \mu}, a^{\prime \nu}\right]\right) \dot{i} \lambda_{A}^{\dot{\alpha}}\left(\bar{\mu}^{A} w_{\dot{\alpha}}+\bar{w}_{\dot{\alpha}} \mu^{A}+\left[a_{\alpha \dot{\alpha}}^{\prime}, M^{\prime \alpha A}\right]\right)\right\} .
\end{aligned}
$$

$\mathcal{S}_{c}^{(k)}$ displays the bosonic and fermionic ADHM constraints.

Consider now correlators of D3/D3 fields, e.g of the scalar $\phi$ in the Cartan direction, in presence of $k$ D-instantons. It turns out [14, 15, 16] that the dominant contribution to $\left\langle\phi_{1} \ldots \phi_{n}\right\rangle$ is from $n$ one-point amplitudes on discs with moduli insertions. The result can therefore be encoded in extra moduli-dependent vertices for $\phi$ 's, i.e. in extra terms in the moduli action containing such one-point functions:

$$
\mathcal{S}_{\text {mod }}(\varphi ; \mathcal{M})=\phi(x) J_{\phi}(\widehat{\mathcal{M}})+\mathcal{S}_{\text {mod }}(\widehat{\mathcal{M}})
$$

where $x$ is the instanton center and $\phi(x) J_{\phi}(\widehat{\mathcal{M}})$ is given by the disc diagrams with boundary (partly) on the $\mathrm{D}(-1)$ 's describing the emission of a $\phi$. To determine $\mathcal{S}_{\bmod }(\phi ; \mathcal{M})$ we systematically compute mixed discs with a scalar $\phi$ emitted from the D3 boundary, such as the one of Fig. 2

Other non-zero diagrams, related by the Ward identities of the susies broken by the $\mathrm{D}(-1)$, couple the other components of the gauge supermultiplet to the moduli. The superfield-dependent moduli action $\mathcal{S}_{\text {mod }}(\Phi ; \mathcal{M})$ is thus obtained by simply letting $\phi(x) \rightarrow \Phi(x, \theta)$.

\section{Inclusion of a graviphoton background}

In the stringy setup, is quite natural to consider also the effect of D-instantons on correlators of fields from the closed string sector. The effect can be encoded in a field-dependent moduli action determined from one-point functions of closed string vertices on instanton discs with moduli insertions. Our aim is to study interactions in the low energy $\mathcal{N}=2$ effective action involving the graviphoton.

The field content of $\mathcal{N}=2$ sugra, namely the metric $h_{\mu \nu}$, the gravitini $\psi_{\mu}^{\alpha A}$ and the graviphoton $C_{\mu}$ can be organized in a chiral Weyl multiplet:

$$
W_{\mu \nu}^{+}(x, \theta)=\mathcal{F}_{\mu \nu}^{+}(x)+\theta \chi_{\mu \nu}^{+}(x)+\frac{1}{2} \theta \sigma^{\lambda \rho} \theta R_{\mu \nu \lambda \rho}^{+}(x)+\cdots
$$




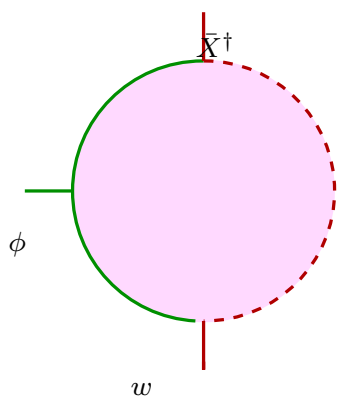

Fig. 2 Emission of a D3 field from a mixed disc.
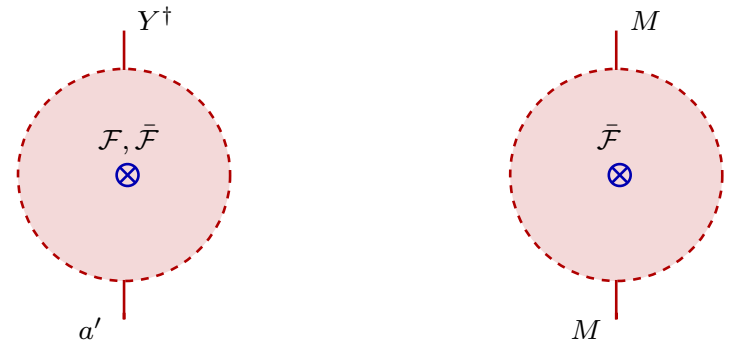

Fig. 3 Graviphoton emission from an instanton disc.

( $\chi_{\mu \nu}^{\alpha A}$ is the gravitino field strength). These fields arise from massless vertices of type IIB strings on $\mathbb{R}^{4} \times \mathbb{C} \times \mathbb{C}^{2} / \mathbb{Z}_{2}$. In particular, the graviphoton vertex is given by]

$$
V_{\mathcal{F}}(z, \bar{z})=\frac{1}{4 \pi} \mathcal{F}^{\alpha \beta A B}(p) \times\left[S_{\alpha}(z) S_{A}(z) \mathrm{e}^{-\frac{1}{2} \varphi(z)} S_{\beta}(\bar{z}) S_{B}(\bar{z}) \mathrm{e}^{-\frac{1}{2} \varphi(\bar{z})}\right] \mathrm{e}^{\mathrm{i} p \cdot X(z, \bar{z})},
$$

left-right movers identification on discs being taken into account. The bi-spinor graviphoton polarization is given by $\mathcal{F}^{(\alpha \beta)[A B]}=\frac{\sqrt{2}}{4} \mathcal{F}_{\mu \nu}^{+}\left(\sigma^{\mu \nu}\right)^{\alpha \beta} \epsilon^{A B}$ and corresponds to a RR 3-form $\mathcal{F}_{\mu \nu z}$ with one "leg" in the $\mathbb{C}$ internal direction. To determine the contribution of the graviphoton to the field-dependent moduli action we have to consider disc amplitudes with open string moduli vertices on the boundary and closed string graviphoton vertices in the interior which survive in the field theory limit $\alpha^{\prime} \rightarrow 0$. Other diagrams, connected by susy, have the effect of promoting the dependence of the moduli action to the full Weyl multiplet: $\mathcal{F}_{\mu \nu}^{+} \rightarrow W_{\mu \nu}^{+}(x, \theta)$. It turns out that very few diagrams (depicted in Fig. 3) contribute.

These diagrams are easily evaluated; for instance, one has

$$
\left\langle V_{M} V_{M} V_{\overline{\mathcal{F}}}\right\rangle=\frac{1}{4 \sqrt{2}} \operatorname{tr}_{k}\left\{M^{\alpha A} M^{\beta B} \overline{\mathcal{F}}_{\mu \nu}^{+}\right\}\left(\sigma^{\mu \nu}\right)_{\alpha \beta} \epsilon_{A B}
$$

Integrating over the moduli the interactions described by the field-dependent moduli action $\mathcal{S}_{\text {mod }}\left(\Phi, W^{+} ; \mathcal{M}(k)\right)$ one gets the effective action, and hence the prepotential, for the long-range multiplets $\Phi$ and $W^{+}$in the instanton number $k$ sector:

$$
S_{\text {eff }}^{(k)}\left[\Phi, W^{+}\right] \equiv \int d^{4} x d^{4} \theta \mathcal{F}^{(k)}\left(\Phi, W^{+}\right)=\int d^{4} x d^{4} \theta d \widehat{\mathcal{M}}_{(k)} \mathrm{e}^{-\frac{8 \pi k}{g^{2}}-\mathcal{S}_{\bmod }\left(\Phi, W^{+} ; \mathcal{M}(k)\right)} .
$$

$\Phi(x, \theta)$ and $W_{\mu \nu}^{+}(x, \theta)$ are constant w.r.t. the integration variables $\widehat{\mathcal{M}}_{(k)}$. We can compute $\mathcal{F}^{(k)}(a ; f)$ giving them constant values: $\Phi(x, \theta) \rightarrow a, W_{\mu \nu}^{+}(x, \theta) \rightarrow f_{\mu \nu}$, getting a "deformed" moduli action

$$
\begin{aligned}
& \mathcal{S}_{\text {mod }}(a, \bar{a} ; f, \bar{f})=S_{\mathrm{c}}^{(k)}-\operatorname{tr}_{k}\left\{\left(\left[\chi^{\dagger}, a_{\alpha \dot{\beta}}^{\prime}\right]+2 \bar{f}_{c}\left(\tau^{c} a^{\prime}\right)_{\alpha \dot{\beta}}\right)\left(\left[\chi, a^{\prime \dot{\beta} \alpha}\right]+2 f_{c}\left(a^{\prime} \tau^{c}\right)^{\dot{\beta} \alpha}\right)\right. \\
& \left.-\left(\chi^{\dagger} \bar{w}_{\dot{\alpha}}-\bar{w}_{\dot{\alpha}} \bar{a}\right)\left(w^{\dot{\alpha}} \chi-a w^{\dot{\alpha}}\right)-\left(\chi \bar{w}_{\dot{\alpha}}-\bar{w}_{\dot{\alpha}} a\right)\left(w^{\dot{\alpha}} \chi^{\dagger}-\bar{a} w^{\dot{\alpha}}\right)\right\} \\
& +\mathrm{i} \frac{\sqrt{2}}{2} \operatorname{tr}_{k}\left\{\bar{\mu}^{A} \epsilon_{A B}\left(\mu^{B} \chi^{\dagger}-\bar{a} \mu^{B}\right)-\frac{1}{2} M^{\alpha A} \epsilon_{A B}\left(\left[\chi^{\dagger}, M_{\alpha}^{B}\right]+2 \bar{f}_{c}\left(\tau^{c}\right)_{\alpha \beta} M^{\beta B}\right)\right\} .
\end{aligned}
$$

Notice that the constraint part of the action, $S_{\mathrm{c}}^{(k)}$, is not modified.

${ }^{1}$ A different RR field, with a similar structure, will be useful ( $\hat{A}, \hat{B}=3,4 \leftrightarrow$ are odd "internal" spin fields):

$$
V_{\overline{\mathcal{F}}}(z, \bar{z})=\frac{1}{4 \pi} \overline{\mathcal{F}}^{\alpha \beta \hat{A} \hat{B}}(p) \times\left[S_{\alpha}(z) S_{\hat{A}}(z) \mathrm{e}^{-\frac{1}{2} \varphi(z)} S_{\beta}(\bar{z}) S_{\hat{B}}(\bar{z}) \mathrm{e}^{-\frac{1}{2} \varphi(\bar{z})}\right] \mathrm{e}^{\mathrm{i} p \cdot X(z, \bar{z})} .
$$


In the action $\mathcal{S}_{\bmod }(a, \bar{a} ; f, \bar{f})$ the v.e.v.'s $a, f$ and $\bar{a}, \bar{f}$ are not on the same footing. Indeed, we have $\mathcal{S}_{\text {mod }}(a, \bar{a} ; f, \bar{f})=Q \Xi$ where $Q$ is the scalar component $Q \equiv \frac{1}{2} \epsilon_{\dot{\alpha} \dot{\beta}} Q^{\dot{\alpha} \dot{\beta}}$ of the twisted supercharges $Q^{\dot{\alpha} B} \stackrel{\text { top.twist }}{\longrightarrow} Q^{\dot{\alpha} \dot{\beta}}$. The parameters $\bar{a}, \bar{f}_{c}$ appear only in the gauge fermion $\Xi$. The instanton partition function

$$
Z^{(k)} \equiv \int d \mathcal{M}_{(k)} \mathrm{e}^{-\mathcal{S}_{\bmod }(a, \bar{a} ; f, \bar{f})}
$$

is therefore independent of $\bar{a}, \bar{f}_{c}$ : its variation w.r.t these parameters is $Q$-exact.

The moduli action obtained inserting the graviphoton background coincides exactly with the "deformed" action [2, 3, 4, 5] considered in the literature to localize the moduli space integration if we set

$$
f_{c}=\frac{\varepsilon}{2} \delta_{3 c}, \bar{f}_{c}=\frac{\bar{\varepsilon}}{2} \delta_{3 c},
$$

and moreover (referring to the notations in the above ref.s) $\varepsilon=\bar{\varepsilon}, \varepsilon=\epsilon_{1}=-\epsilon_{2}$. From the explicit form of $\mathcal{S}_{\text {mod }}(a, 0 ; f, 0)$ it follows that the prepotential $\mathcal{F}^{(k)}(a ; f)$ is invariant under $a, f_{\mu \nu} \rightarrow-a,-f_{\mu \nu}$. It must moreover be regular for $f \rightarrow 0$, to reproduce the instanton \# $k$ contribution to the SW prepotential. Since odd powers of $a f_{\mu \nu}$ cannot appear, one has altogether, reinstating the superfields,

$$
\mathcal{F}^{(k)}\left(\Phi, W^{+}\right)=\sum_{h=0}^{\infty} c_{k, h} \Phi^{2}\left(\frac{\Lambda}{\Phi}\right)^{4 k}\left(\frac{W^{+}}{\Phi}\right)^{2 h}
$$

Summing over the instanton sectors we obtain

$$
\mathcal{F}_{\text {n.p. }}\left(\Phi, W^{+}\right)=\sum_{k=1}^{\infty} \mathcal{F}^{(k)}\left(\Phi, W^{+}\right)=\sum_{h=0}^{\infty} C_{h}(\Lambda, \Phi)\left(W^{+}\right)^{2 h}, C_{h}(\Lambda, \Phi)=\sum_{k=1}^{\infty} c_{k, h} \frac{\Lambda^{4 k}}{\Phi^{4 k+2 h-2}} .
$$

This yields many different terms in the effective action, connected by susy. In particular, saturating the $\theta$ integration with four $\theta$ 's all from $W^{+}$we get

$$
\int d^{4} x C_{h}(\Lambda, \phi)\left(R^{+}\right)^{2}\left(\mathcal{F}^{+}\right)^{2 h-2} .
$$

To compute $c_{k, h}$, one can use constant values $\Phi \rightarrow a$ and $W_{\mu \nu}^{+} \rightarrow f_{\mu \nu}$; we will use the values in Eq. 22 that correspond to the localization deformation.

As we remarked above, $Z^{(k)}(a, \varepsilon)$ does not depend on $\bar{\varepsilon}$. However, $\bar{\varepsilon}=0$ is a limiting case: some care is needed. In fact, while $\mathcal{F}^{(k)}(a ; \varepsilon)$ is well-defined, $S^{(k)}[a ; \varepsilon]$ diverges because of the (super)volume integral $\int d^{4} x d^{4} \theta$. The presence of $\bar{\varepsilon}$ regularizes the superspace integration by a Gaussian term, leading to the effective rule: $\int d^{4} x d^{4} \theta \rightarrow 1 / \varepsilon^{2}$; one can then work with the effective action, i.e., the full instanton partition function. Moreover, $a$ and $\varepsilon, \bar{\varepsilon}$ deformations localize completely the integration over moduli space which can thus be carried out [2, 3, 4, 5].

With $\bar{\varepsilon} \neq 0$ (complete localization) a trivial superposition of instantons of charges $k_{i}$ contributes to the sector $k=\sum k_{i}$; such disconnected configurations do not contribute when $\bar{\varepsilon}=0$. The partition function computed by localization corresponds thus to the exponential of the non-perturbative prepotential:

$$
Z(a ; \varepsilon)=\exp \left(\frac{\mathcal{F}_{\text {n.p. }}(a, \varepsilon)}{\varepsilon^{2}}\right)=\exp \left(\sum_{k=1}^{\infty} \frac{\mathcal{F}^{(k)}(a, \varepsilon)}{\varepsilon^{2}}\right)=\exp \left(\sum_{h=0}^{\infty} \sum_{k=1}^{\infty} c_{k, h} \frac{\varepsilon^{2 h-2}}{a^{2 h}}\left(\frac{\Lambda}{a}\right)^{4 k}\right) .
$$

In conclusion, the computation via localization techniques of the multi-instanton partition function $Z(a ; \varepsilon)$ determines the coefficients $c_{k, h}$ which appear in the gravitational $F$-terms of the $\mathcal{N}=2$ effective action Eq. (4) via the expression of $C_{h}(\Lambda, \phi)$ given in Eq. (3).

The very same gravitational $F$-terms can been extracted in a completely different way by considering topological string amplitudes on suitable Calabi-Yau manifolds. Indeed, the construction that goes under 
the name of geometrical engineering embeds directly the $S W$ low energy description of $\mathcal{N}=2 \mathrm{SYM}$ theory into string theory as type IIB on a suitable local CY manifold $\mathfrak{M}$ [6, 7]. In this realization, the geometric moduli of $\mathfrak{M}$ encode the gauge theory data $(\Lambda, a)$, and the coefficients $C_{h}$ in the 1.e.e.a. gravitational F-terms are given by topological string amplitudes at genus $h$ [8, 9].

The two different roads to determine the $F$-couplings of Eq. (4) must lead to the same result. This is a very natural way to state the conjecture by $\mathrm{N}$. Nekrasov [2] that the coefficients arising in the $\varepsilon$-expansion of multi-instanton partition functions match those appearing in higher genus topological string amplitudes on CY manifolds.

Acknowledgements I thank the organizers of the Workshop "ForcesUniverse 2006" and the co-authors of [1]. Work partially supported by the E.C. Human Potential Programme, contract MRTN-CT-2004-005104, and by the Italian MIUR, contract PRIN-2005023102.

\section{References}

[1] M. Billo, M. Frau, F. Fucito and A. Lerda, JHEP 0611 (2006) 012 [arXiv:hep-th/0606013].

[2] N. A. Nekrasov, Adv. Theor. Math. Phys. 7 (2004) 831 [arXiv:hep-th/0206161].

[3] R. Flume and R. Poghossian, Int. J. Mod. Phys. A 18 (2003) 2541 |arXiv:hep-th/0208176].

[4] A. S. Losev, A. Marshakov and N. A. Nekrasov, |arXiv:hep-th/0302191|; N. Nekrasov and A. Okounkov, [arXiv:hep-th/0306238].

[5] N. A. Nekrasov, Class. Quant. Grav. 22 (2005) S77.

[6] S. Kachru, A. Klemm, W. Lerche, P. Mayr and C. Vafa, Nucl. Phys. B 459, 537 (1996) |arXiv:hep-th/9508155]; A. Klemm, W. Lerche, P. Mayr, C. Vafa and N. P. Warner, Nucl. Phys. B 477, 746 (1996) |arXiv:hep-th/9604034].

[7] S. Katz, A. Klemm and C. Vafa, Nucl. Phys. B 497, 173 (1997) [arXiv:hep-th/9609239].

[8] M. Bershadsky, S. Cecotti, H. Ooguri and C. Vafa, Nucl. Phys. B 405 (1993) 279 [arXiv:hep-th/9302103]; Commun. Math. Phys. 165 (1994) 311 [arXiv:hep-th/9309140].

[9] I. Antoniadis, E. Gava, K. S. Narain and T. R. Taylor, Nucl. Phys. B 413 (1994) 162 [arXiv:hep-th/9307158].

[10] A. Klemm, M. Marino and S. Theisen, JHEP 0303 (2003) 051 |arXiv:hep-th/0211216|.

[11] E. Witten, Nucl. Phys. B 460 (1996) 335 [arXiv:hep-th/9510135].

[12] M. R. Douglas, J. Geom. Phys. 28, 255 (1998) |arXiv:hep-th/9604198]; arXiv:hep-th/9512077

[13] J. Polchinski, Phys. Rev. D 50 (1994) 6041 [arXiv:hep-th/9407031|.

[14] M.B. Green and M. Gutperle, Nucl. Phys. B 498 (1997) 195, |arXiv:hep-th/9701093|; JHEP 9801 (1998) 005, |arXiv:hep-th/9711107]; Phys. Rev. D 58 (1998) 046007, [arXiv:hep-th/9804123].

[15] M.B. Green and M. Gutperle, JHEP 0002 (2000) 014 [arXiv:hep-th/0002011].

[16] M. Billo, M. Frau, I. Pesando, F. Fucito, A. Lerda and A. Liccardo, JHEP 0302 (2003) 045 [arXiv:hep-th/0211250]. 